\title{
Link Dimensioning in WDM Networks with Dynamic Traffic Grooming
}

\author{
K. Mosharaf, I. Lambadaris, J. Talim, and A. Shokrani \\ Department of Systems and Computer Engineering, Carleton University \\ Emails:\{mosharaf,jtalim,ioannis,shokrani\}@sce.carleton.ca
}

\begin{abstract}
This paper presents an analytical model to determine the number of required wavelengths to meet a specified Grade of Service (GoS) in WDM networks with dynamic grooming capabilities. The GoS is defined in terms of overall call blocking probability in the system. Traffic consists of two rate streams, low-rate and high-rate. For each traffic stream, call interarrival and holding times are exponentially distributed. Two wavelength assignment strategies, Random and FirstFit, are considered to accommodate the calls in the network. In the case of Random assignment, the system can be formulated as a Markov process from which a product form solution can be approximated the overall call blocking probabilities. Numerical and simulation results show that in low traffic regime this formula is a lower bound for the Random assignment and an upper bound for the First-Fit assignment. Numerically, we show that the product form formula can be used to dimension the network, when implementing the First-Fit assignment strategy.
\end{abstract}

Keywords - Wavelength Division Multiplexing (WDM), Markov Process, Link Dimensioning, Blocking Performance.

\section{Introduction}

Wavelength Division Multiplexing (WDM), using wavelength routing, is one of the candidates to handle the huge bandwidth of future wide area backbone networks. Although each wavelength can have a bandwidth up to tens of gigabit per second (e.g., OC-48 or OC-192), some traffic streams still need lower bandwidth (e.g., OC-12 or OC-48). In order to utilize the wavelengths more efficiently, traffic grooming is deployed in WDM networks. Optical Add/Drop Multiplexers (OADMs) can perform the traffic grooming by multiplexing/demultiplexing the low-rate traffic streams onto the wavelengths [1]. The traffic grooming problem can be formulated as a TDM problem. Therefore, the bandwidth of each wavelength may be divided into a set of time slots. For instance, if the wavelength capacity is OC-48, then different combinations of sessions with the OC-12 and OC48 rates can be accommodated in the same wavelength. Therefore, each wavelength can be subdivided into 4 slots of length OC-12. Consequently, in order to establish a call in the system, calls with rates of OC-12 and OC-48 need 1 and 4 time-slots, respectively.

Many heuristic algorithms such as First-Fit, Most-Used, MaxSum and Random wavelength assignment have already been proposed for the case when each call occupies the whole wavelength [2]. The objective of these algorithms is typically to minimize the overall call blocking probabilities in a single-rate WDM network. Some complementary analytical models related to this problem can be found in [3], [4]. Some more general models for multi-rate WDM networks are discussed in [5], [6]. In [5], a capacity correlation model is proposed for a multi-hop single wavelength path, whereas [6] discusses the numerical computation of the blocking probabilities in a two-class network, without capacity correlation between the links.
In this paper, we present an analytical model to evaluate the blocking performance for a multi-wavelength multi-rate network while considering the correlation between slot occupancies. We assume that there are high-rate streams and low-rate streams of calls. The objective is to design an algorithm to determine the required number of wavelengths in order to meet a specified GoS. When implementing the Random assignment strategy, the system can be modeled in an appropriate manner as a Markov Chain which has a product-form solution. Nevertheless, this solution may be inefficient because of its computational complexity, even for moderate sized networks. One way to reduce the numerical complexity is to develop approximations and study the behavior of the system in different regimes [7], [8]. Hence, we will develop approximations and investigate the asymptotic behavior of the blocking probabilities.

The rest of the paper is organized as follows: In Section 2, we formulate the problem as a Markov process and propose an approximate solution to compute the blocking probabilities. Section 3 deals with the asymptotic behavior of the system for low load traffic and Section 4 presents the performance of the proposed method when we use it to dimension the network. Conclusions will be presented in Section 5.

\section{Problem Formulation}

Consider a single-hop multiple-wavelength path for a circuitswitched wavelength routing network. Assume that each link carries one fiber with $W$ wavelengths and each wavelength consists of $T$ time slots. Traffic is divided into 2 classes: Class $c_{1}$ requires $t_{1}$ time-slots, $t_{1}<T$, to be established; Class $c_{2}$ carries streams with time-slot requirement $t_{2}, t_{2}=T$. Therefore, a class $c_{2}$ call is accepted whenever there is at least one complete wavelength available. Each call must be entirely accommodated in one wavelength and can not split among wavelengths. Any arriving call from either class is blocked when no wavelength has enough free space to accommodate it. Blocked calls do not interfere with the system. For each class, call interarrival times and holding times are exponentially distributed. Random wavelength assignment and fixed routing is assumed.

We also define the following notation.

- $n_{i}^{j}, i=1,2, j=1,2, . ., W$, is the number of class $c_{i}$ calls currently carried by wavelength $j$.

- $\lambda_{i}^{-1}$, is the mean interarrival time of class $c_{i}$ calls.

- $\mu_{i}^{-1}$, is the mean holding time of class $c_{i}$ calls.

In order to distribute the resources among the users in a fair manner, we assume that $t_{1} \lambda_{1}=t_{2} \lambda_{2}$ and their respective mean holding times are equal to unity. Hence, the expected time-slot request rates of the two classes are the same.

We consider first a simple model with two wavelengths. The problem can be modeled as a Markov Chain and the system 

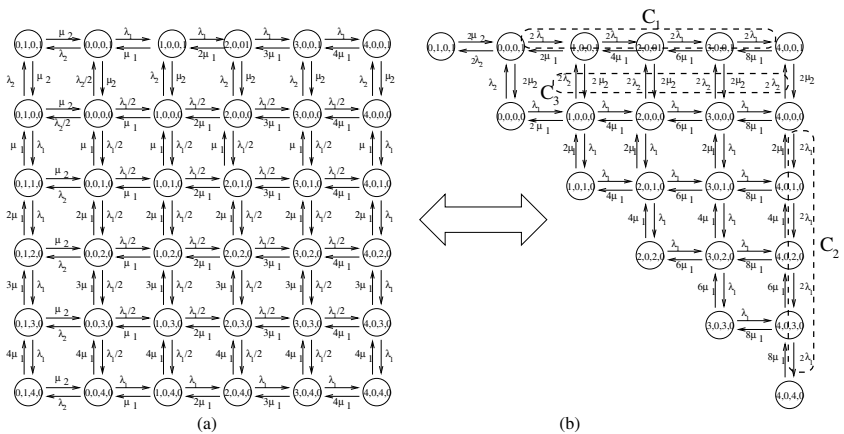

Figure 1. Markov Chain Representation of the system with $W=2, t_{1}=1, t_{2}=T=4$. (a) The original Model, (b) The simplified model after considering symmetric property

state can be characterized by a four-component vector $s=$ $\left(n_{1}^{1}, n_{2}^{1}, n_{1}^{2}, n_{2}^{2}\right)$. The state space in this case can be expressed as:

$$
\begin{array}{r}
\Omega_{2} \equiv\left\{\left(n_{1}^{1}, n_{2}^{1}, n_{1}^{2}, n_{2}^{2}\right) \in \mathbb{N}^{4}\right. \\
\left.t_{1} n_{1}^{1}+t_{2} n_{2}^{1} \leq T, \quad t_{1} n_{1}^{2}+t_{2} n_{2}^{2} \leq T\right\}
\end{array}
$$

Let $s_{t}$ be the system state at time $t$. Then $\left\{s_{t}, t \geq 0\right\}$ is a Markov Chain (MC) from which we can compute the stationary slot occupancy probabilities. Fig. 1(a) depicts the MC of the simple problem with $W=2, t_{1}=1, t_{2}=T=4$. According to this figure, one can observe the symmetric structure of the MC. Due to the symmetric nature of the MC shown in Fig. 1(a), it can be easily shown that the stationary probability of this MC can be determined from the stationary probability of the simpler MC depicted in Fig. 1(b). Thus, instead of solving the MC showed in Fig. 1(a), we can determine the stationary slot occupancy probabilities, and in particular the blocking probabilities, from a simpler MC depicted in Fig. 1(b). Note that all transition rates are twice as large as the ones in Fig. 1(a).

In order to calculate the probabilities for the MC in Fig. 1(b), we consider the reversibility criterion or the Kolomogorov's criterion [9]:

$$
\begin{aligned}
& t_{r}\left(s_{1} \rightarrow s_{2}\right) t_{r}\left(s_{2} \rightarrow s_{3}\right) \cdots t_{r}\left(s_{n} \rightarrow s_{1}\right)= \\
& t_{r}\left(s_{1} \rightarrow s_{n}\right) t_{r}\left(s_{n} \rightarrow s_{n-1}\right) \cdots t_{r}\left(s_{2} \rightarrow s_{1}\right)
\end{aligned}
$$

where $t_{r}\left(s_{i} \rightarrow s_{j}\right)$ is the transition rate from state $s_{i}$ to state $s_{j}$. It shows that cyclic paths are bidirectional and the overall transition rate along a cycle is the same in either direction.

From Fig. 1(b), we can see that some cycles such as the cycle connecting the set of states:

$$
\{(0,0,0,0),(1,0,0,0),(1,0,0,1),(0,0,0,1)\}
$$

do not meet the reversibility criterion. Thus, the system is not time-reversible and does not have the product form solution.

To overcome this problem, if we modify some of the transition rates in a way that the $\mathrm{MC}$ becomes time-reversible, then we can obtain the product from solution for the modified time-reversible MC. We introduce the following transition rate modifications to the MC depicted in Fig. 1(b):

$\begin{cases}t_{r}\left(\left(n_{1}^{1}, 0,0,1\right) \rightarrow\left(n_{1}^{1}+1,0,0,1\right)\right)=\lambda_{1} & \text { if } n_{1}^{1}=0,1,2,3 \\ t_{r}\left(\left(4,0, n_{1}^{2}, 0\right) \rightarrow\left(4,0, n_{1}^{2}+1,1\right)\right)=\lambda_{1} & \text { if } n_{1}^{2}=0,1,2,3 \\ t_{r}\left(\left(n_{1}^{1}, 0,0,0\right) \rightarrow\left(n_{1}^{1}, 0,0,1\right)\right)=\lambda_{2} & \text { if } n_{1}^{1}=0,1,2,3\end{cases}$

The modified MC is time-reversible and has the following product form solution:

$$
\pi(s)=\frac{1}{G(2)} \times \frac{\rho_{1}^{\left(n_{1}^{1}+n_{1}^{2}\right)} \rho_{2}^{\left(n_{2}^{1}+n_{2}^{2}\right)}}{n_{1}^{1} ! n_{2}^{1} ! n_{1}^{2} ! n_{2}^{2} ! \times 2^{\left(n_{1}^{1}+n_{2}^{1}+n_{1}^{2}+n_{2}^{2}\right)}}
$$

where $s=\left(n_{1}^{1}, n_{2}^{1}, n_{1}^{2}, n_{2}^{2}\right), \rho_{i}=\lambda_{i} / \mu_{i}$ is the traffic intensity of class $c_{i}$ stream, $\pi(s)$ is the probability that the system state is $s$ and $G(2)$ is the normalizing constant.

Fig. 1(a) shows that, for $W=2$, the system can be modeled by a 2-dimensional MC. The generalization of the analysis to $W>2$ wavelengths yields Markov Chains of $W$ dimensions with the following state space:

$$
\Omega_{W} \equiv\left\{n_{1}^{j}, n_{2}^{j} \geq 0, t_{1} n_{1}^{j}+t_{2} n_{2}^{j} \leq T, j=1,2, \ldots, W\right\}
$$

Because of the Random assignment strategy, the symmetry of this MC still holds. Using the same idea as before, we can extract a 2-dimensional truncated MC from the original $W$-dimensional MC based on the symmetry, whose transition rates are $W$ times as large as the ones of the original MC.

Similar to (3), we can modify the transition rates of the truncated MC so that the final modified 2-dimensional MC becomes time-reversible. The following equation gives the product form solution in steady state for a network with $W$ wavelengths:

$$
\pi(s)=\frac{1}{G(W)} \prod_{j=1}^{W} \frac{\rho_{1}^{n_{1}^{j}} \times \rho_{2}^{n_{2}^{j}}}{n_{1}^{j} ! \times n_{2}^{j} ! \times W^{\left(n_{1}^{j}+n_{2}^{j}\right)}} \text { for } s \in \Omega_{W}
$$

where $s=\left(n_{1}^{1}, n_{2}^{1}, n_{1}^{2}, n_{2}^{2}, \ldots, n_{1}^{W}, n_{2}^{W}\right), \pi(s)$ is the probability that the system state is $s$ and the normalizing constant, $G(W)$, is:

$$
G(W)=\sum_{s \in \Omega_{W}}\left(\prod_{j=1}^{W} \frac{\rho_{1}^{n_{1}^{j}} \times \rho_{2}^{n_{2}^{j}}}{n_{1}^{j} ! \times n_{2}^{j} ! \times W^{\left(n_{1}^{j}+n_{2}^{j}\right)}}\right)
$$

\section{Asymptotic Behavior for Light Load Traffic}

In this section, we study the behavior of the system while it is fed with light traffic. One can note that from (5), the cardinality of $\Omega_{W}$ is given by $6^{W}$. Therefore, the calculation of the normalizing constant is no longer trivial and requires considerable computation, when the number of wavelengths increases.

Our objective is to determine the minimum number of wavelengths to satisfy the GoS, where the GoS is characterized by the overall blocking probability which we assume takes a small 
value, for example $10^{-3}$. Thus, we can restrict our analysis to a system carrying light traffic, or equivalently $\rho_{2} / W<$ $\rho_{1} / W<<1$. Using this assumption, we can say that the term $\left(\frac{\rho_{1}}{W}\right)^{n_{1}^{j}} / n_{1}^{j}$ !, for $n_{1}^{j}>1$, is negligible when compared to $\rho_{1} / W$. Accordingly, it may be omitted in the calculation of $G(W)$. Similarly, term $\left(\frac{\rho_{2}}{W}\right)^{n_{2}^{j}} / n_{2}^{j}$ !, for $n_{2}^{j}>1$, can be neglected.

In this part, we show the approximation of $G(W)$, for the example shown in Fig. 1. Using (7), let write $G(2)$ as:

$$
\begin{array}{r}
G(2)=\sum_{s \in \Omega_{2}} \frac{\left(\frac{\rho_{1}}{2}\right)^{n_{1}^{1}} \cdot\left(\frac{\rho_{2}}{2}\right)^{n_{2}^{1}}}{n_{1}^{1} ! \cdot n_{2}^{1} !} \times \frac{\left(\frac{\rho_{1}}{2}\right)^{n_{1}^{2}} \cdot\left(\frac{\rho_{2}}{2}\right)^{n_{2}^{2}}}{n_{1}^{2} ! \cdot n_{2}^{2} !} \\
=\underbrace{\sum_{\left\{n_{1}^{2}+4 n_{2}^{2} \leq 4\right\}} \frac{\left(\frac{\rho_{1}}{2}\right)^{n_{1}^{2}} \cdot\left(\frac{\rho_{2}}{2}\right)^{n_{2}^{2}}}{n_{1}^{2} ! \cdot n_{2}^{2} !}}_{\sum_{k=1, \cdots 4} \frac{\left(\frac{\rho_{1}}{2}\right)^{k}}{k !} \cdot I+\left(\frac{\rho_{2}}{2}\right) \cdot I}+
\end{array}
$$

In (8), we can remove the terms $\frac{\left(\frac{\rho_{1}}{2}\right)^{k}}{k !}$ for $k>1$. Hence, we can rewrite (8) as:

$$
G(2) \simeq I \times\left(1+\frac{\rho_{1}}{2}+\frac{\rho_{2}}{2}\right)
$$

We now decompose $I$ by considering the possible values of $\left(n_{1}^{2}, n_{2}^{2}\right)$ so that $\left(n_{1}^{2}+4 n_{2}^{2} \leq 4\right)$

$$
I=1+\frac{\frac{\rho_{1}}{2}}{1 !}+\frac{\left(\frac{\rho_{1}}{2}\right)^{2}}{2 !}+\frac{\left(\frac{\rho_{1}}{2}\right)^{3}}{3 !}+\frac{\left(\frac{\rho_{1}}{2}\right)^{4}}{4 !}+\frac{\frac{\rho_{2}}{2}}{1 !} \simeq 1+\frac{\rho_{1}}{2}+\frac{\rho_{2}}{2}
$$

We derive a simple approximation of $G(2)$ :

$$
G(2) \simeq\left(1+\frac{\rho_{1}}{2}+\frac{\rho_{2}}{2}\right)^{2}
$$

Using the assumption $\frac{\rho_{1}}{2}+\frac{\rho_{2}}{2}<<1$ and the fact that $e^{x} \simeq 1+x$ for $x<<1$, we conclude:

$$
G(2) \simeq e^{\rho_{1}+\rho_{2}}
$$

In general we can show that $G(W) \simeq e^{\rho_{1}+\rho_{2}}$ for low load regime and consequently, the stationary probability $\pi(s)$ in (6) can be approximated by:

$$
\pi(s) \simeq \frac{1}{e^{\left(\rho_{1}+\rho_{2}\right)}} \prod_{j=1}^{W} \frac{\left(\frac{\rho_{1}}{W}\right)^{n_{1}^{j}} \times\left(\frac{\rho_{2}}{W}\right)^{n_{2}^{j}}}{n_{1}^{j} ! \times n_{2}^{j} !}
$$

The above equation estimates the product form solution under the asymptotic condition for low load traffic. It shows that in this regime, we do not need to compute the normalizing constant.

\section{Performance Evaluation}

This section is devoted to the numerical computation of the blocking probabilities (BP). As shown in [2], the First-Fit wavelength assignment scheme is a simple and efficient candidate to apply in WDM networks. For comparison, we implement

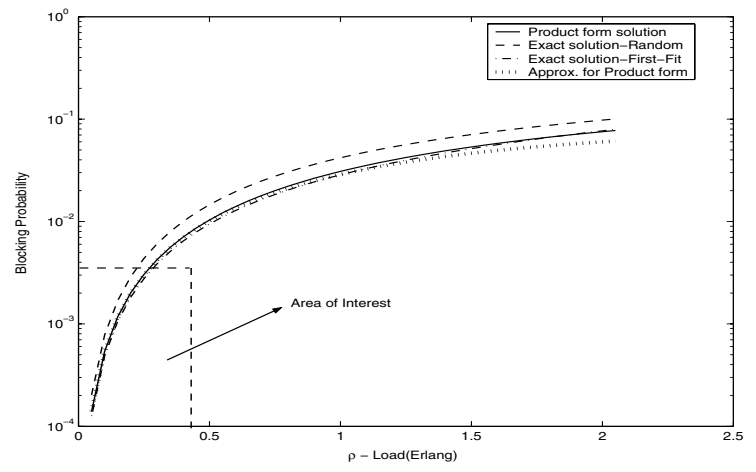

Figure 2: Overall blocking probabilities for a 2-wavelength link

both the Random and First-Fit assignment schemes and compare them with the results of section 2 and 3 .

For the case of two wavelengths when deploying the Random assignment scheme, we use the generator matrix $Q^{*}$ to compute the stationary probability for the MC shown in Fig. 2(a). The following equations lead us to calculate the exact solution for the Random assignment.

$$
\pi(s) Q^{*}=0, \pi(s) e=1
$$

We applied the above numerical method to calculate the exact blocking probabilities for First-Fit assignment as well. We plot the exact blocking probabilities as functions of the traffic load $\left(\rho=\rho_{1}+\rho_{2}\right)$ for the Random and First-Fit policies and compare them with the ones computed by equations (4) and (13). Fig. 2 illustrates that the First-Fit policy has better performance than the Random specially for the low load traffic. It also depicts that the closed form solution (4) is a lower bound for the Random assignment. In the area of interest, the solution by equation (4) is an almost indistinguishable upper bound of the First-Fit assignment and the result from (13) yields a very good approximation of (4).

To evaluate a 3-wavelength system, we simulate the system using OPNET, because the numerical solutions are not easy to derive. Fig. 3 shows the simulation results for this case. The accuracy of the approximations, as mentioned previously, holds

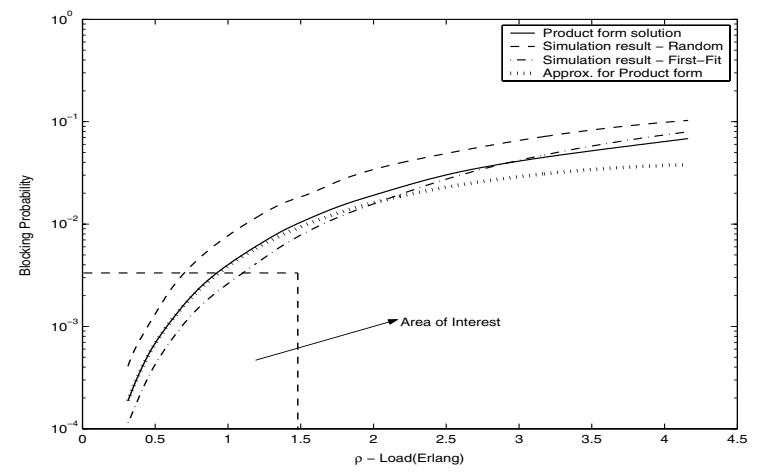

Figure 3: Overall blocking probabilities for 3-wavelength link 


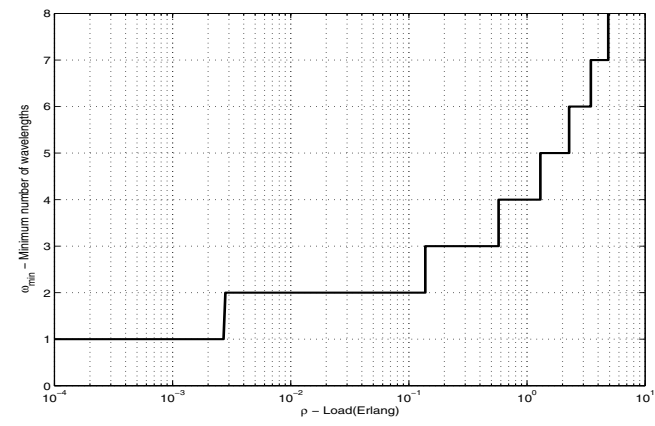

Figure 4: Minimum number of wavelengths to satisfy $\mathbf{G o S}=10^{-3}$

for the 3-wavelength system.

Since the First-Fit assignment provides better performance in terms of utilization and blocking probabilities (see Figs. 2, 3), it is more likely to be the policy implemented in practice. For a GoS of the order $10^{-3}$ or $10^{-5}$, the formula (13) is a good approximation of the experienced blocking probability when the First-Fit assignment is used. Therefore, we use (13) to determine $W_{\min }$, the minimum number of wavelengths to meet a specified GoS.

We plot $W_{\min }$ in Fig. 4 as a function of the total offered traffic load in logarithm scale. Fig. 5 provides the comparison of $W_{\min }$ for two GoSs, GoS $=10^{-3}$ and $\mathrm{GoS}=10^{-5}$. Let $\rho$ be the total offered traffic, and $f$ is a mapping: $f: \rho \mapsto W_{\text {min }}$. Fig. 5 illustrates that:

- As $\rho$ increases, the slope of $f$ decreases and the average traffic carried per wavelength increases. This is referred to as the economies of scale. For $\mathrm{GoS}=10^{-3}$, the system requires 8 wavelengths to accommodate a total traffic of 5 Erlang. Thus, in the average a wavelength carries $(5 / 8) \sim 0.625$ traffic load in Erlang. For the same GoS, if we increase the traffic load to 35 Erlang, then we only need 21 wavelengths. In this case, each wavelength carries $(35 / 21) \sim 1.67$ Erlang traffic load.

- By increasing GoS from $10^{-3}$ to $10^{-5}$ for the same load, the slope of $f$ increases as well. This shows that a system with more stringent GoS is more demanding in terms of resources.

- In addition, we also investigate a mathematical interpolation model of the mapping $f$ that provides the minimum number of

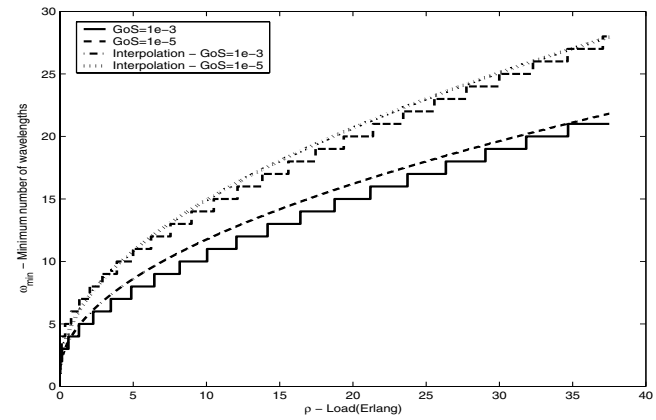

Figure 5. Minimum number of wavelengths to satisfy $\mathrm{GoS}=10^{-3}$ and $\mathrm{GoS}=10^{-5}$ wavelengths required for a given total load and a given GoS. For $\mathrm{GoS}=10^{-3}$ (respectively, $\mathrm{GoS}=10^{-5}$ ), our mathematical interpolation is $W_{\min }(\rho)=1+3.4 \sqrt{\rho}$ (respectively, $W_{\min }(\rho)=$ $1+4.4 \sqrt{\rho})$.

\section{Conclusions}

We have proposed an approach for network dimensioning in WDM networks with dynamic grooming capabilities. The problem has been formulated as a Markov process from which an approximate product form expression of the overall call blocking probabilities was derived. We use this formula to determine the minimum number of wavelengths required to meet a specified GoS. The simulation results show that our proposed method provides a very good estimation of the call blocking probabilities in system while realistic First-Fit assignment policy is used. Therefore, it may be used to determine the number of wavelengths to dimension a WDM network. We are currently working on the problem of converting the number of wavelengths into the number of optical fibers per link, with the objective of minimizing the cost of fibers as well as the cost of OADMs.

\section{References}

[1] K. Zang and B. Mukherjee, "A Review of Traffic Grooming in WDM optical networks: Architectures and Challenges," Optical Networks Magazine, vol. 4, no. 2, Mar./Apr. 2003, pp. 55-64.

[2] H. Zang, J. P. Jue, and B. Mukherjee, "A Review of Routing and Wavelength Assignment Approaches for Wavelength-Routed Optical WDM Networks," Optical Networks Magazine, vol. 1, no. 1, Jan. 2000, pp. 47-60

[3] R. A. Barry and P. A. Humblet, "Models of Blocking Probability in All-Optical Networks with and without Wavelength Changers," IEEE J. Sel. Areas Comm., vol. 14,no. 5, June 1996, pp. 858-867.

[4] A. Birman, "Computing Approximate Blocking Probabilites for a Class of All-Optical Networks," IEEE J. Sel. Areas Comm., vol. 14,no. 5, June 1996, pp. 852-857.

[5] S. Thiagarajan and A. K. Somani, "A Capacity Correlation Model for WDM Networks with Constrainted Grooming Capabilities," Proc. IEEE ICC 2001, June 2001, pp. 15921596.

[6] V. Tamilraj and S. Subramaniam, "An Analytical Blocking Model for Dual-Rate Sessions in Multichannel Optical Networks," Proc. IEEE GLOBECOM 2001, Nov. 2001, pp. 1515-1519.

[7] P. Gazdziki, I. Lambadaris and R. Mazumdar, , 25, 1993. "Blocking Probabilities for Large Multirate Erlang Loss System," Journal of Advances in Applied Probability, vol. 25, Dec. 1993, pp. 997-1009.

[8] J. P. Labourdette and G. W. Hart "Blocking Probabilities in Multitraffic Loss Systems: Insensivity, Asymptotic Behavior, and Approximation," IEEE Trans. on Comm., vol. 40, no. 8, Aug. 1992.

[9] F. P. Kelly, "Reversibility and Stochastic Networks," John Wiley and Sons, New York, 1979. 